\title{
Permethrin exposure affects neurobehavior and cellular characterization in rats' brain
}

\author{
Gabriel Omotoso ${ }^{1 *}$ (D), Olajumoke Oloyede ${ }^{1}$, Shakirah Lawal ${ }^{1}$, Ismail Gbadamosi 1, Nafisat Mutholib 1, Fatimah \\ Abdulsalam 1, Abdulkabir Bature 1, Abdulsalam Babalola ${ }^{1}$, Busola Ayeni ${ }^{1}$, and Nathaniel Amedu ${ }^{1}$ \\ ${ }^{1}$ Department of Anatomy, Faculty of Basic Medical Sciences, University of Ilorin, P.M.B. 1515, Ilorin, Nigeria \\ *Correspondence: omotoso.go@unilorin.edu.ng, gabrielolaiya@yahoo.com
}

Received: June 11, 2020 Accepted: October 13, 2020

\begin{abstract}
This study investigated the neurotoxic effects of permethrin on the cerebellum, hippocampus and prefrontal cortex of Wistar rats and its effects on some behavioral patterns. Fifteen adult male Wistar rats were grouped into three categories: Group A received $0.1 \mathrm{~mL}$ normal saline (control), and Groups B and C received mixed feed with $500 \mathrm{mg} / \mathrm{kg}$ and 1,000 $\mathrm{mg} / \mathrm{kg}$ of $0.6 \%$ permethrin, respectively, for 14 days. The animals were assessed for memory, anxiety and exploratory locomotion and thereafter anesthetized and transcardially perfused with normal saline and $4 \%$ paraformaldehyde (PFA). Cerebellum, hippocampus and prefrontal cortex were excised from the whole brain and processed for tissue histology, histochemistry and immunohistochemistry. Oxidative status and lipid peroxidation were also assessed using catalase, glutathione peroxidase, superoxide dismutase and malondialdehyde as biomarkers. Results revealed dosedependent decrease in body weights but increase in cerebellar and prefrontal weights, depletion of endogenous antioxidant markers, cognitive deficits, reduced locomotor activities, degenerative changes in the microarchitecture at high doses and presence of chromatolytic cells at both low and high doses of permethrin. Astrocytes were activated while synaptophysin expression was downregulated. Permethrin causes dose-dependent neurotoxicity on the morphology, neurochemistry and oxidative status of different brain regions, and these could affect behavioral performance and other neurologic functions.
\end{abstract}

Keywords: permethrin, behavior, brain morphology, neurotoxicity, oxidative stress

\section{Introduction}

Permethrin is a synthetic pyrethroid insecticide derived from natural pyrethrins from the plant Chrysanthemum cinerariaefolium [1]. They are commonly used for the control of insect pests and disease vectors [2]. Permethrin can be used in the treatment of lice and scabies, as pet shampoos, in treating wood furniture and can be applied on the surface of clothing and mosquito nets $[3,4]$. Permethrin-treated clothing serves as a form of personal protection to prevent bites from disease vectors such as mosquitoes and ticks [5,6] and has been used in the fight against malaria [7]. In the agricultural sector, permethrin is used in pest control to protect agricultural products such as cotton, wheat and maize [2].

Exposure to permethrin could be via inhalation, topical or by eating contaminated food items, and when in excess, can cause nausea, headache, muscle weakness, excessive salivation and breathing problems $[1,8,9]$. Although absorption following topical exposure is minimal compared to other modes of absorption, the side effects vary depending on the nature of the skin and the dose of permethrin exposed to. Previous studies showed that $0.5 \%$ absorption occurred in the first 48 hours [9], and highest amounts of permethrin accumulate in fats and the brain [10]. The lipophilic nature of the permethrin molecule is a factor in this regard. Permethrin is metabolized in the liver, where it undergoes oxidation by the cytochrome P450 system, as well as hydrolysis into various metabolites [9].

Pyrethroids share the same mode of action [4] and are capable of crossing the blood-brain barrier to exert their effects on the brain thereby causing neurotoxicity and motor deficits [11, 12]. Their insecticidal action targets voltage-gated sodium channels and this mechanism has also been implicated in the neurotoxicity associated with pyrethroids use in mammals, although other channels such as the voltage-gated calcium and chloride channels are also considered important [2]. Their action makes the channels stay open much longer than normal, leading to hyper-excitation of the central nervous system [13]. Insects have more sensitive voltage-gated sodium channels, making them to be much more sensitive to pyrethroids than higher animals [14].

Permethrin is neurotoxic at elevated doses and its various adverse effects have been associated with its exposure in animal studies, including tremor, salivation, paresthesia, depressed reflexes and peripheral nerve damage [13, 15]. Early exposure in life is detrimental to brain development with the likelihood of the occurrence and persistence of a lifelong 
disability. Nasuti et al. [4], observed that neonatal permethrin exposure leads to long-term cognitive impairment and alteration in synaptic morphology. In view of the risk involved in permethrin exposure and its neurotoxic potential, this study was embarked upon to ascertain the cellular, molecular and behavioral characterization associated with permethrin neurotoxicity.

\section{Materials and Methods}

Laboratory animals and care

A total of 15 adult male Wistar rats weighing between 130-135 g were used for the study. The study was conducted in the Animal House of the Central Research Laboratory, University of Ilorin, Nigeria. The animals were allowed to acclimatize for two weeks prior to commencement of the study, and they had access to feeds and water liberally under hygienic conditions.

\section{Treatment of animals}

Rambo insect powder (Rambo®; Gongoni Co. Ltd, Kano, Nigeria) containing $0.6 \%$ Permethrin and $99.4 \%$ inert carriers was procured and used for the study. The animals were divided into three groups (A - C) each consisting of five rats. Group A received standard rat diet; Group B received standard rat diet mixed with $500 \mathrm{mg} / \mathrm{kg}$ permethrin insecticide; Group C received standard rat diet mixed with 1,000 mg/kg permethrin insecticide [16]. Treatment was for 14 consecutive days.

\section{Behavioral study}

After the last day of treatment, the rats were assessed for memory, learning, exploratory activities, anxiety, and locomotion.

\section{Morris water maze test}

Morris water maze test was carried out to assess spatial learning and memory of the rats [17]. A pool of water in a container measuring about $100 \mathrm{~cm}$ in diameter and $30 \mathrm{~cm}$ in depth was used. An escape platform about $2.5 \mathrm{~cm}$ deep from the surface of the water was placed in one of the quadrants and visual cues were provided. The animals were trained 24 hours before the main test was conducted. During the training period, each rat was placed in each of the other three quadrants for a maximum of 60 seconds to find the escape platform at an interval of 15 minutes between quadrants until the escape latency period reduced to less than 15 seconds. During the real test, the pool was colored and the animals were placed in each of the three quadrants different from the escape platform quadrant at an interval of 15 minutes between quadrants. The time taken to find the escape platform was noted as the escape latency period.

\section{Y-maze test}

Y-maze was used to examine the working memory of the rats [18]. The animals were placed in a Y-maze with arms measuring $75 \mathrm{~cm}$ in length and $15 \mathrm{~cm}$ in breath with an angle of $120^{\circ}$ in between arms. The animals were allowed to explore the maze for duration of five minutes. The manner of alternation was recorded. The percentage correct alternation of each rat was estimated as a ratio of the correct alternation to the total alternation multiplied by 100.

\section{Open field test}

The open field apparatus was made from plywood $(100 \mathrm{~cm} \times 100 \mathrm{~cm} \times 50 \mathrm{~cm})$ [19]. The floor was divided into square grids each measuring $25 \mathrm{~cm}$ in length with a blue marker and a center square of the same length was drawn with a red marker. During the test, the rats were picked by their tails and dropped in the center square and allowed to explore for five minutes while the video was captured by a camera. The five behavioral parameters scored were: number of lines crossed, center square entry, center square duration, rearing frequency and stretch attend posture. The number of lines crossed was the frequency with which the rats crossed one of the grid lines with all four paws; the center square entry was the frequency with which the rats crossed one of the red lines with all four paws into the central square while the center square duration was the total time spent in the center square. The rearing frequency was the number of times the animal stood on its hind limbs, while the stretch attend postures was the frequency with which the animal demonstrated forward elongation of the head and shoulders followed by retraction to the original position.

\section{Black and white box test}

This is a behavioral paradigm used for testing anxiety [20]. The fine wooden apparatus $(75 \mathrm{~cm} \times 30 \mathrm{~cm} \times 30 \mathrm{~cm})$ was divided into a light chamber ( $50 \mathrm{~cm}$ long) and a dark chamber $(25 \mathrm{~cm}$ long). The light and dark chambers were connected by a small passage through which the animal can move freely. The light chamber was illuminated with natural white light 
while the dark chamber was cove and not illuminated. During the test, the rat was placed in the center of the light chamber, facing the opening of the dark chamber. The animals were allowed an exploration time of 10 minutes; thereafter the apparatus was cleaned to remove debris that may be left by the previous animal. The total time spent in the dark chamber was estimated and subjected to statistical analysis.

\section{Negative geotaxis test}

The geotaxis test [21] is used to investigate motor coordination and vestibular sensitivity in rodents. The negative geotaxis is made up of an inclined surface with grids at $45^{\circ}$. The rats were placed on the inclined grid in the head downwards position. The grid allows for grip and permits the rodent to reorient itself towards an upwards position. The time taken for the animal to reorient itself to an upward direction was recorded as latency of turning.

\section{Tissue processing for histological, histochemical and immunohistochemical demonstration}

After termination of study, the animals for qualitative analysis (2 rats per group) were anesthetized intramuscularly with $0.5 \mathrm{~mL} / \mathrm{kg}$-ketamine. They were subjected to transcardial perfusion with $50 \mathrm{~mL}$ normal saline, followed by $4 \%$ paraformaldehyde (PFA). After perfusion, the animals were decapitated in the cervical region. The brain tissues were excised (cerebellum, hippocampus and prefrontal cortex), weighed and fixed in $4 \%$ PFA for 48 hours. The tissues were processed for histology, embedded in paraffin and sectioned at $5 \mu \mathrm{m}$ thick with the aid of a Rotary microtome. Tissue staining was carried out using hematoxylin and eosin (H\&E) stain for general histology and cresyl fast violet (CFV) stain for histochemical demonstration of Nissl substances [22, 23]. For the immunohistochemical study, serial sections (10 $\mu \mathrm{m})$ were taken from paraffin blocks, with protein cross-linkages removed in the sections by applying $0.1 \%$ trypsin to retrieve the antigens. Hydrogen peroxide was used to block endogenous peroxidase, while $5 \%$ bovine serum albumin (BSA) was used to reduce nonspecific protein reactions. Primary antibodies (anti-glial fibrillary acidic protein (GFAP) and antisynaptophysin) dilution was done in blocking buffer (10\% calf serum with $1 \%$ BSA and $0.1 \%$ Triton X-100 in 0.1 M PBS [phosphate buffered saline]): both were diluted at 1:100. Following this, secondary biotinylated antibody was desalted and diluted in PBS ( $\mathrm{pH}$ 8.0) prior to its application on tissue sections. Incubation with secondary antibody was done in the humidity chamber. Immunogenic reaction was developed using 3,3'-diaminobenzidine and intensified using methenamine silver kit (used according to the manufacturer's instruction). The sections were counterstained in hematoxylin and subsequently treated in $1 \%$ acid alcohol to reduce the counterstain intensity [24].

\section{Colorimetric assay for biochemical studies}

Rats meant for enzymatic studies (3 rats per group) were sacrificed by cervical dislocation without tissue perfusion with PFA. Assay kits for superoxide dimustase (SOD; KT-60703), catalase (CAT; MBS701713), glutathione peroxidase (GPx; MBS744364) and malondialdehyde (MDA; MBS9389391) were used to assess the cerebellum, hippocampus and prefrontal cortex of rats using spectrophotometric technique [25]. All reagents and samples were set to room temperature before starting the procedures. Equal weights of brain tissues were homogenized in ice cold $30 \%$ sucrose solution with an automated homogenizer at $4^{\circ} \mathrm{C}$. The homogenate was scooped and poured into a $5 \mathrm{~mL}$ plain specimen bottle and placed in a centrifuging tube containing ice. The homogenate was centrifuged for 15 minutes at 3,000 rpm. The supernatants were aspirated into plain labeled glass cuvettes placed in ice. Assay was carried out according to the manufacturer's instruction on the assay pack.

\section{Light microscopy, cell count and statistical analysis}

Histological, histochemical and immunohistochemical sections were captured using Olympus binocular research microscope (Olympus, New Jersey, USA) which was connected to a 5.0 MP Amscope Camera (Amscope Inc, USA). Protein expression and histochemical labelling count were determined using Image J (NIH, USA).

\section{Data analysis}

Data obtained were analyzed and subjected to statistical analysis using the version 16.0 of the Statistical Package for Social Sciences (SPSS). Data were presented as mean \pm SEM (standard error of mean) with determination of level of significance at $p$ values less than 0.05 or 0.01 . The results obtained from the SPSS software were presented in bar charts with error bars, using the 10.0 version of the GraphPad application.

\section{Results}

\section{Permethrin induced loss of body weight, but increased brain weights}

Observation of the body weights of experimental animals revealed that permethrin consumption led to progressive reduction in weights in a dose-dependent fashion, with rats given the higher dose having the least body weight change (Table 1). The opposite occurred for the organ weights of prefrontal cortex and cerebellum, where the brain weights 
increased with increasing dose of permethrin. However, hippocampal weight was higher in rats treated with the low dose compared to those treated with high dose of permethrin. Only cerebellar weight increase in the high dose permethrin group was statistically significant when compared with the control $(\mathrm{p}<0.05)$.

\section{Permethrin differentially affects memory, spatial exploration with no effects on anxiety and locomotion}

We subjected rats to five behavioral paradigms to obtain information on the effects of varied doses of permethrin on spatial memory, working memory, spatial navigation, anxiety and locomotion indices. Extrapolations from the Morris water maze and Y-maze behavioral paradigms showed that permethrin had differential affectation on the spatial memory and working memory respectively (Figure 1). We noted that the higher the dose of permethrin the poorer the memory indices in both behavioral paradigms. However, it was only the high dose permethrin group that had a significantly higher escape latency period in the Morris water maze (MWM) and significantly lower percentage correct alternation in the Y-maze relative to the control group $(\mathrm{p}<0.05)$. This finding suggests that excessive exposure to permethrin has memory dwindling effect. In furtherance, spatial navigation indices in the open field behavioral paradigm reduced as permethrin dose increased. The control rats had a higher frequency of entry into the center square and rearing when compared to the permethrintreated rats $(\mathrm{p}<0.05)$. Further analysis of data obtained from open field behavioral paradigm, black and white box, and negative geotaxis showed that permethrin had no locomotory or anxiety-related effects. There was no significant difference in time spent in dark chamber, freezing duration, latency of turning and number of lines crossed in the permethrin-treated group relative to the control group $(\mathrm{p}>0.05)$.

\section{Effects of permethrin on oxidative stress markers and lipid peroxidation}

The activities of SOD, CAT and GPx as markers of oxidative stress were quantified in the prefrontal cortex, hippocampus and cerebellum. Permethrin consumption decreased the activities of SOD and GPx across the three aforementioned brain regions despite no significant difference ( $p>0.05)$. However, permethrin treatment significantly altered the activities of catalase in all brain regions; high dose permethrin had significantly lower catalase activities in the prefrontal cortex and cerebellum relative to the control group $(\mathrm{p}<0.05)$. Both low dose and high dose permethrin presented with significantly reduced catalase activities in the hippocampus relative to the control group ( $<<0.05)$. MDA was used as a marker of lipid peroxidation. The analysis showed that high dose permethrin induced lipid peroxidation in the prefrontal cortex as the level of MDA in this brain region was significantly higher compared with the control ( $<<0.05)$. Although MDA levels in hippocampal and cerebellar homogenates increased with increasing permethrin dosage, this was not statistically significant $(\mathrm{p}>0.05)$ (Figure 2).

\section{Effects of permethrin exposure on histoarchitecture and Nissl profile of prefrontal cortex, dentate gyrus of hippocampus and cerebellum}

Observation of the prefrontal cortex, hippocampus and cerebellum showed apparently normal histomorphology in the control rats (Figure 3). The prefrontal cortex showed external granular layer with fine granule cells with intact neuropil and deeply stained nuclei. Permethrin-treated rats revealed mild cortical alterations with the presence of increased perinuclear spaces in the low dose group and reduced cellular density in the high dose group. The dentate gyrus of the control group showed a densely packed granular layer transiting into the outer molecular layer characterized by cellular paucity. Despite the striking similarities between the histoarchitectural presentations of the dentate gyrus of the permethrintreated groups with that of the control, there were fragmentations in the histology of the permethrin-treated groups. The cerebellar cortex of the control and low dose group showed that the granular and molecular layers were well delineated by the Purkinje layer. However, the Purkinje layer of the cerebellar cortex of rats treated with high permethrin dose was almost completely obliterated, with marked degeneration of Purkinje cells, leaving a widened space between the granular and

Table 1. Body and brain weights

\begin{tabular}{ccccccc}
\hline Groups & $\begin{array}{c}\text { Final body } \\
\text { weight (g) }\end{array}$ & $\begin{array}{c}\text { Initial body } \\
\text { weight (g) }\end{array}$ & $\begin{array}{c}\text { Body weight } \\
\text { difference (g) }\end{array}$ & $\begin{array}{c}\text { Prefrontal cortical } \\
\text { weight (g) }\end{array}$ & $\begin{array}{c}\text { Hippocampal } \\
\text { weight (g) }\end{array}$ & $\begin{array}{c}\text { Cerebellar } \\
\text { weight (g) }\end{array}$ \\
\hline Control & $159.6 \pm 0.4$ & $134.6 \pm 0.4$ & 25.0 & $0.253 \pm 0.028$ & $0.426 \pm 0.054$ & $0.20 \pm 0.1725$ \\
\hline $\begin{array}{c}\text { Permethrin } \\
(500 \mathrm{mg} / \mathrm{kg})\end{array}$ & $156.2 \pm 0.2$ & $136.2 \pm 0.2$ & 20.0 & $0.281 \pm 0.075$ & $0.506 \pm 0.031$ & $0.39 \pm 0.0876$ \\
\hline $\begin{array}{c}\text { Permethrin } \\
(1000 \mathrm{mg} / \mathrm{kg})\end{array}$ & $153.4 \pm 0.4$ & $135.0 \pm 0.4$ & 18.4 & $0.355 \pm 0.072$ & $0.438 \pm 0.077$ & $0.49 \pm 0.0296^{*}$ \\
\hline
\end{tabular}

*Statistically significant increase in cerebellar weight compared with control $(\mathrm{p}<0.05)$. Data express as mean \pm standard error $(\mathrm{SEM})(\mathrm{n}=5)$. 
molecular layers. Furthermore, Nissl profiling of the three brain areas was assessed. Permethrin induced chromatolytic changes in all the three brain regions examined. High dose permethrin led to the development of central chromatolysis in the pyramidal cells of the prefrontal cortex, granule cells of the dentate gyrus and the Purkinje cells of the cerebellum (Figure 4). This finding suggested that high dose permethrin treatment has chromatolytic effects which may progress to cell death and neurodegeneration if the neurotoxicant was not withdrawn (Figure 5 and Figure 6A).
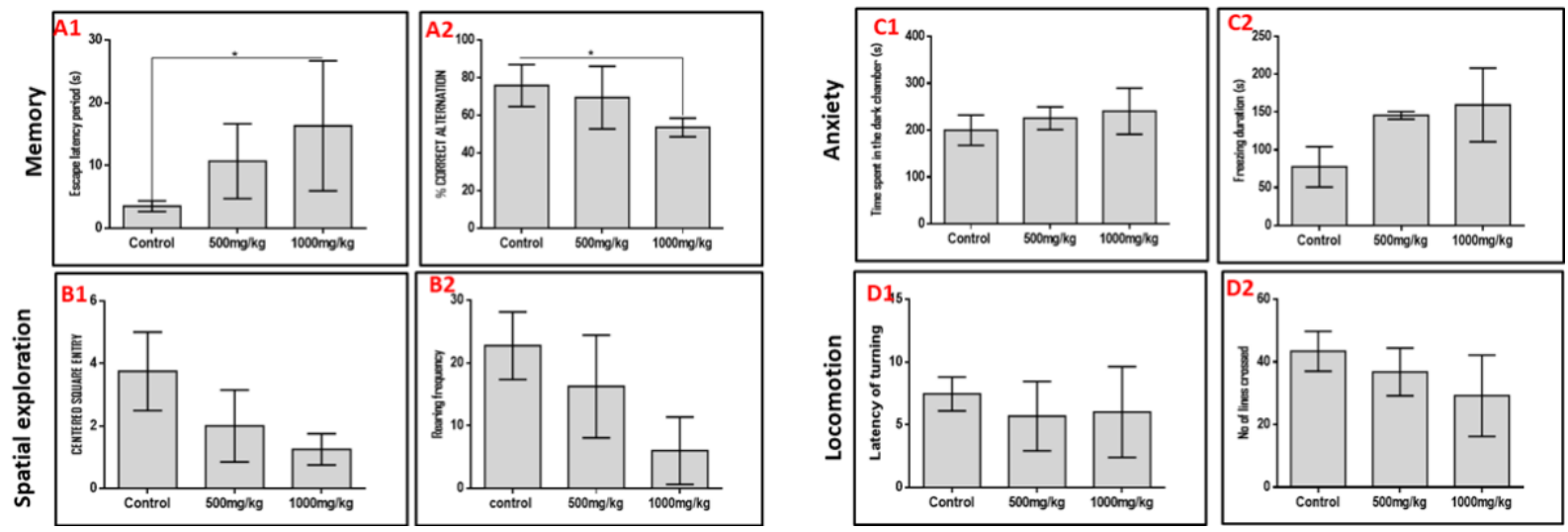

Figure 1. Outcome of behavioral analyses of animals after being exposed. Behavioral paradigm of memory (A1: escape latency period, and A2: \% correct alternation), spatial exploration (B1: centered square entry, and B2: rearing frequency), anxiety (C1: time spent in the dark chamber, and C2: freezing duration) and locomotion (D1: latency of turning, and D2: number of lines crossed). High dose of permethrin (1000 mg/kg) significantly increased the escape latency period and reduced the \% correct alternation relative to the control group in the Morris water maze and Y maze, respectively.

${ }^{*} \mathrm{p}<0.05$.

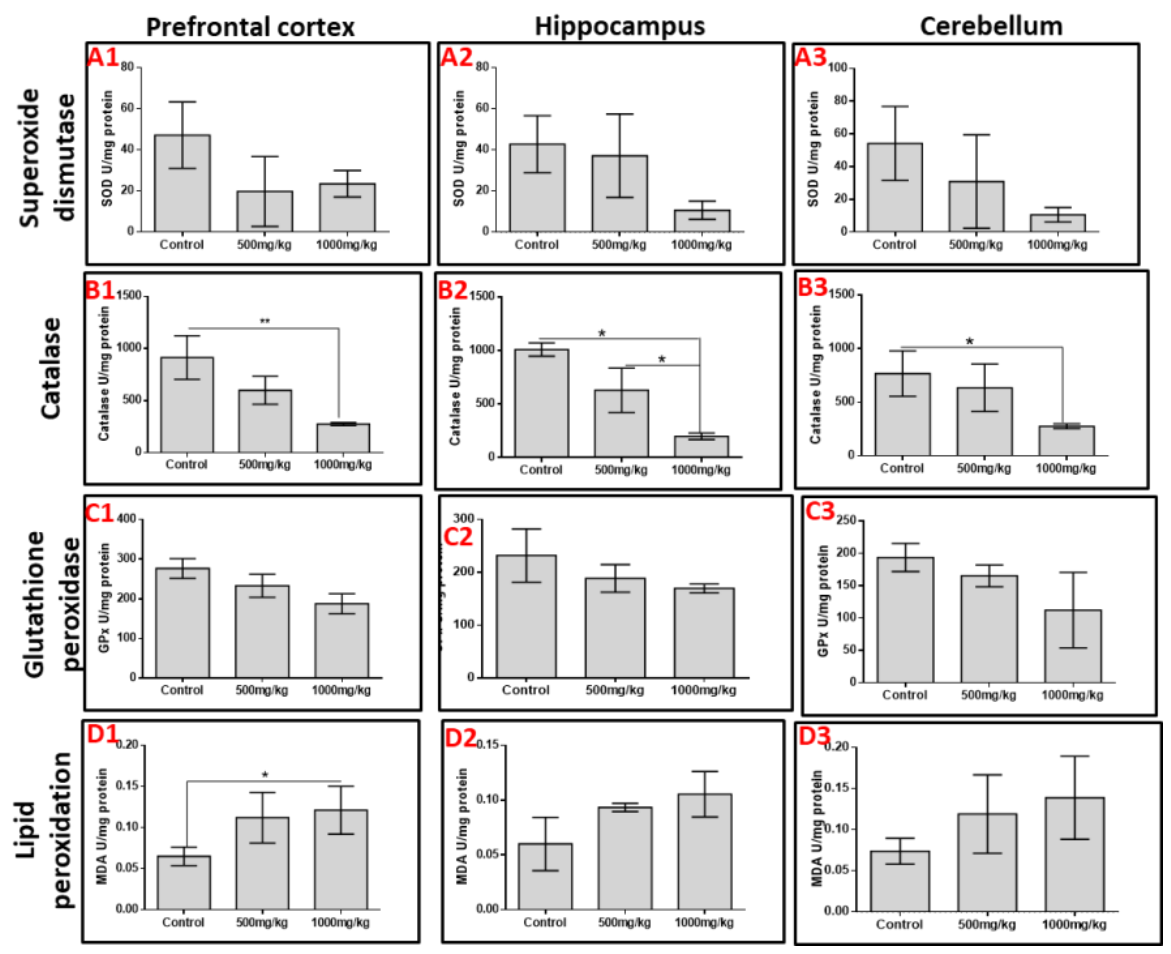

Figure 2. Activities of superoxide dismutase (SOD; A1-A3), catalase (CAT; B1-B3), glutathione peroxidase (GPx; C1-C3) and lipid peroxidation using malon-dialdehyde (MDA; D1-D3) in the prefrontal cortex, hippo-campus and cerebellum. Permethrin showed dose-dependent affectation of the activities of these enzymes in almost all parts of the brain. Permethrin significantly depleted the levels of CAT in all three brain regions when compared to the control while increasing MDA in the prefrontal cortex.

${ }^{*} \mathrm{p}<0.05,{ }^{* *} \mathrm{p}<0.01$. 


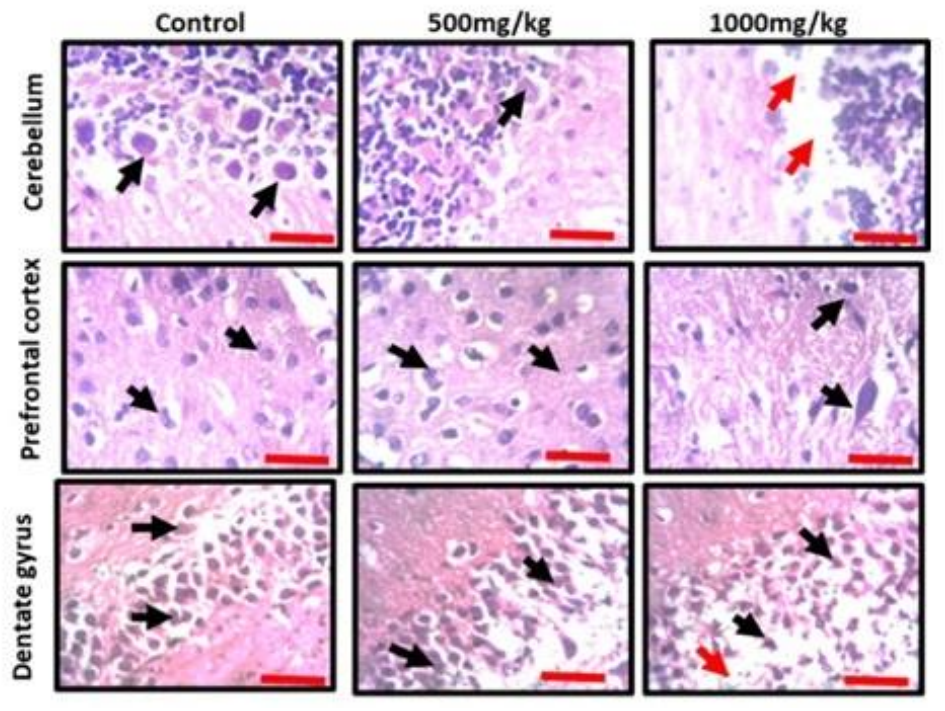

Figure 3. Representative photomicrographs of the cerebellum, prefrontal cortex and hippocampus of animals that received standard diet (control), low dose permethrin diet $(500 \mathrm{mg} / \mathrm{kg}$ ) and high dose permethrin diet (1000 $\mathrm{mg} / \mathrm{kg}$ ). The control presented with the typical histomorphology of the three brain regions. The cerebellar cortex showed densely packed internal granular layer separated from the external molecular layer by a single-celled layer of Purkinje cells (black arrow) in the control section, while the low dose group revealed a reduced staining intensity of Purkinje cells. The Purkinje layer was markedly destroyed in the high dose group, with possible absence of Purkinje cells (red arrows), and the cellular density in the molecular layer was reduced. The prefrontal cortex showed external granular layer made up of fine granule cells (black arrow), with increased perinuclear spaces in the low dose group and reduction in cell population in the high dose group. The dentate gyrus of the hippocampus revealed densely and finely packed granule cells (control; black arrow), however, cellular density reduced in permethrin-treated rats with increased intercellular spaces, and cellular arrangement was disorganized especially in the high dose group (Hematoxylin and Eosin; Scale bar: $25 \mu \mathrm{m}$ ).

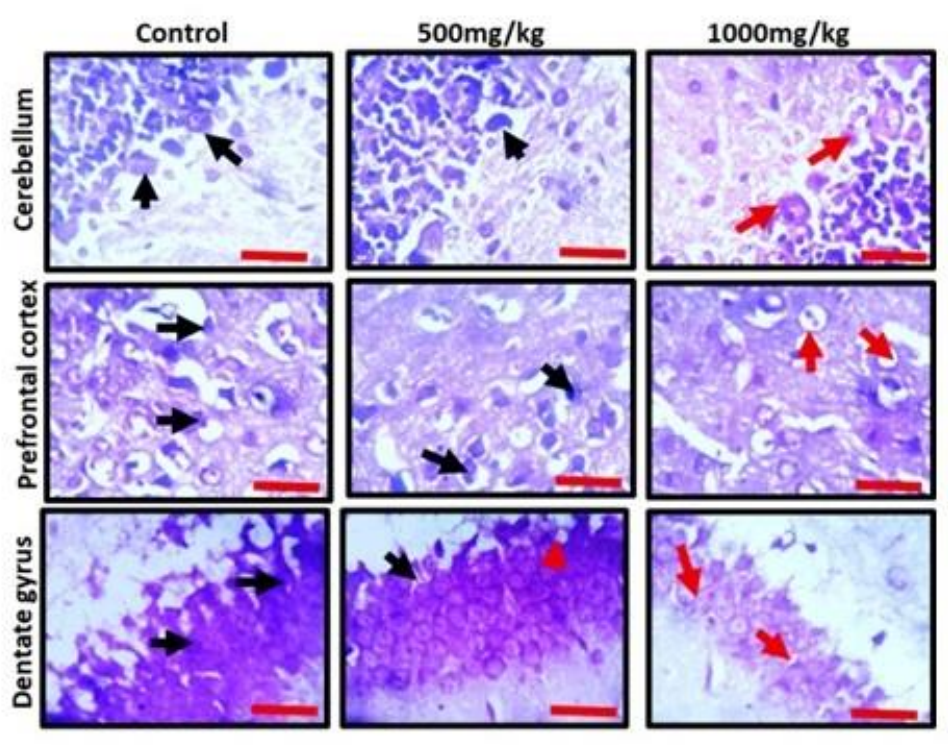

Figure 4. Representative photomicrographs of Nissl profile of cerebellum, prefrontal cortex and dentate gyrus of experimental animals treated with standard diet (control), low dose permethrin $(500 \mathrm{mg} / \mathrm{kg})$ and high dose permethrin $(1000 \mathrm{mg} / \mathrm{kg})$. The control animals presented with typical Nissl staining intensity across the three brain regions. The neurons were deeply stained (black arrows) and well situated in their respective neuropils. The low dose and high dose treated groups presented with chromatolytic cells (red arrows). The dentate gyrus of the high dose group particularly appeared poorly stained (Cresyl fast violet; Scale bar is $25 \mu \mathrm{m}$ ). 


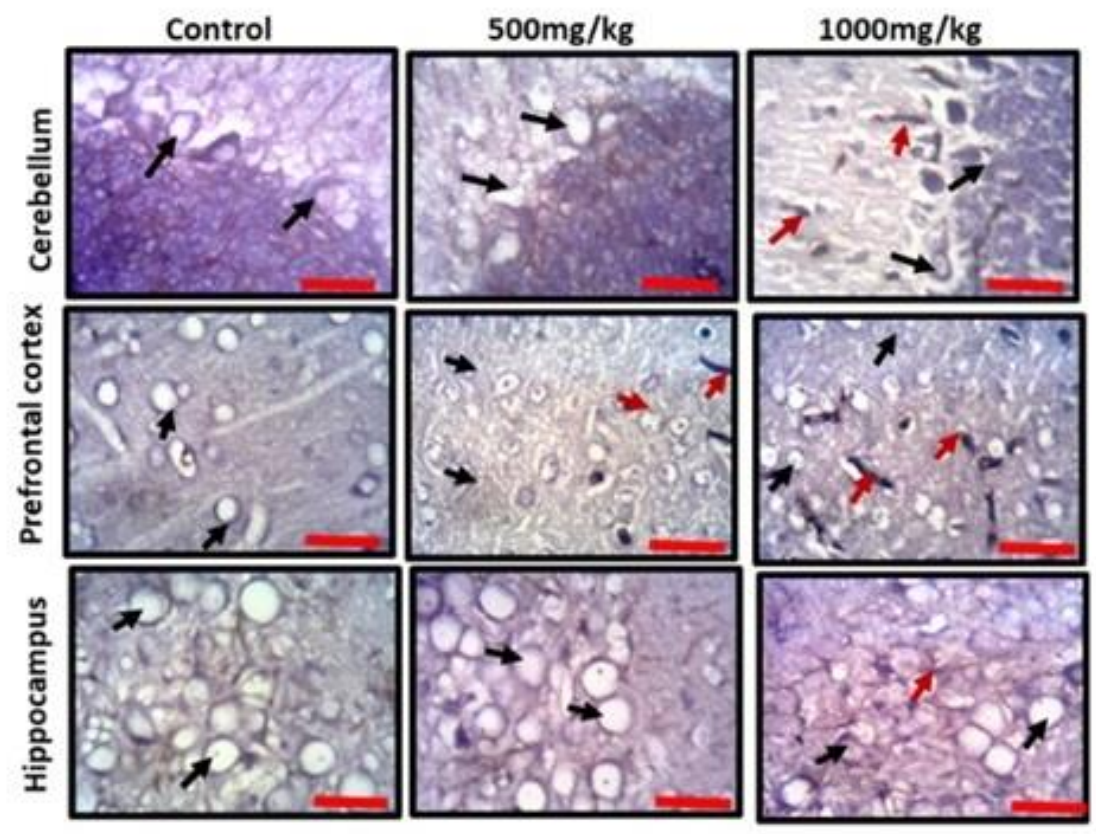

Figure 5. Representative photomicrographs of the cerebellum, prefrontal cortex and hippocampus of experimental animals treated with standard diet (control), low dose permethrin $(500 \mathrm{mg} / \mathrm{kg})$ and high dose permethrin $(1000 \mathrm{mg} / \mathrm{kg})$ showing anti-GFAP immunopositivity (red arrow) and nuclei of inherent neurons as counterstained by hematoxylin (black arrow) (scale bar: $25 \mu \mathrm{m}$ ).

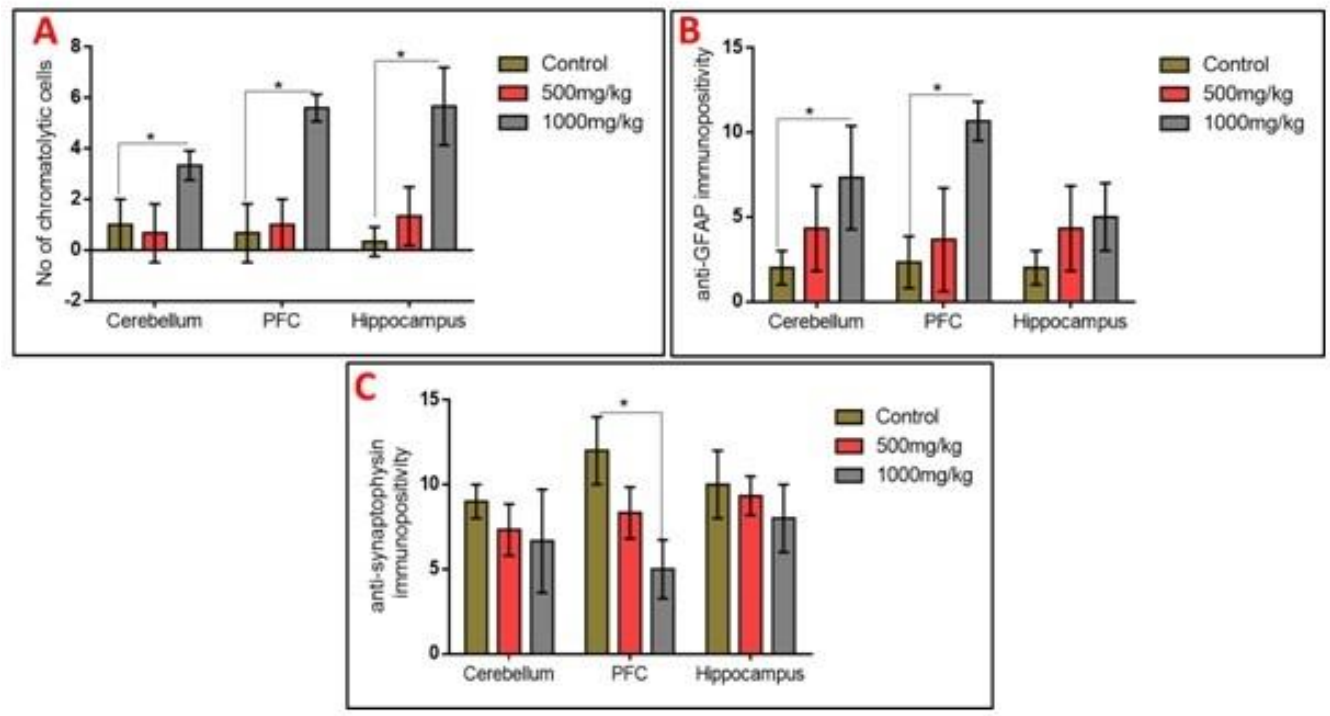

Figure 6. Cell counting using Image-J software. A, B and C represented Nissl profile, anti-GFAP immunopositivity and anti-synaptophysin immune-positivity, respectively. ${ }^{*} \mathrm{p}<0.05$.

Glial activation and reduced expression of the synaptic vesicular protein in the prefrontal cortex, hippocampus and cerebellum following permethrin exposure

To further elucidate the possible effects of permethrin on the aforementioned three brain regions, we characterized activated astroglia and synaptophysin. High dose permethrin significantly increased the expression of activated astroglial cells. The hypertrophied immunopositive cells were well expressed in the molecular layer of the cerebellum as well as the external pyramidal layer of the prefrontal cortex. Following automated cell counting, anti-GFAP immunopositivity in the 


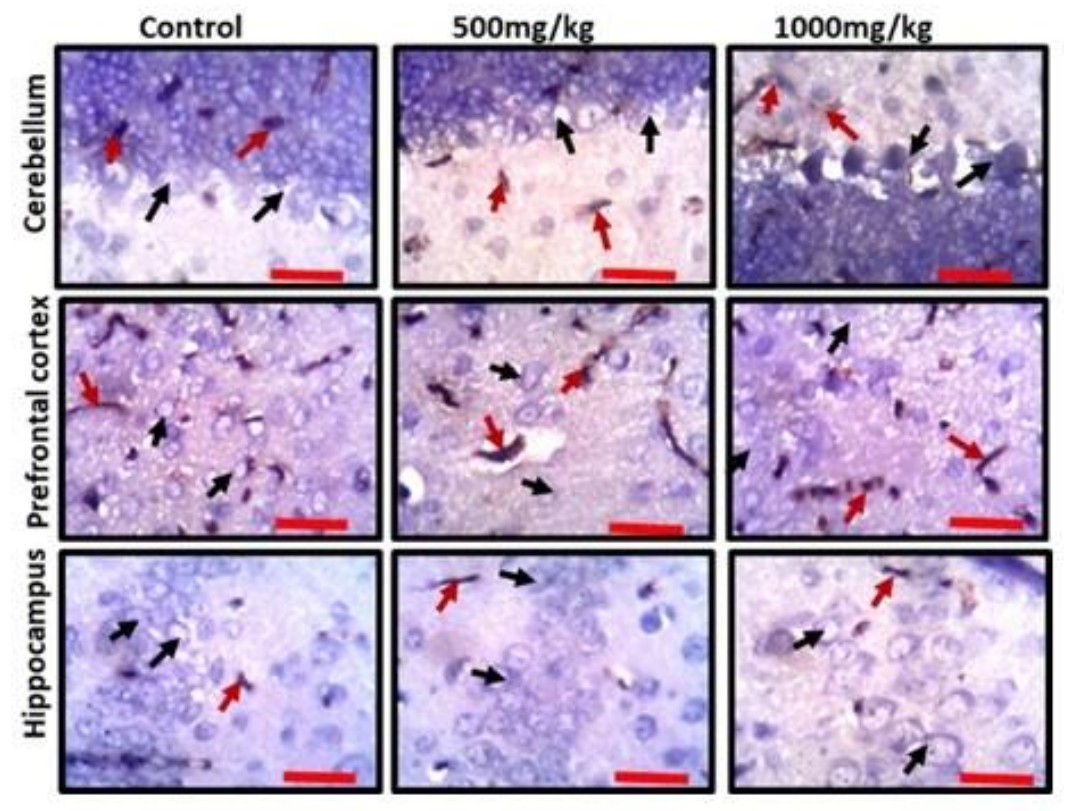

Figure 7. Representative photomicrographs of the cerebellum, prefrontal cortex and hippocampus of experimental animals treated with standard diet (control), low dose permethrin $(500 \mathrm{mg} / \mathrm{kg}$ ) and high dose permethrin $(1000 \mathrm{mg} / \mathrm{kg})$ showing anti-synaptophysin immunopositivity (red arrow) and nuclei of other neuronal cells as counterstained by hematoxylin (black arrow). (Scale bar: $25 \mu \mathrm{m}$ )

hippocampus of permethrin-treated rats was not significantly high, unlike in the prefrontal and cerebellar cortices where there was a significant increase in the expression of these cells (Figure 5 and Figure 6B). Synaptophysin is a structural protein expressed by vesicles at synaptic terminals; hence, a pathological modification or downregulation of this protein will result in the compromise of structural and functional integrity of synaptic transport vesicles. Analysis of expression of this synaptic protein showed an inverse relationship between synaptophysin and dose of permethrin such that the cellular immunopositivity reduced as the dose increased. However, following automated synaptophysin polarity, there was dosedependent reduction in immunopositive cells across the groups, but this was not statistically significant ( $p>0.05)$ (Figure 6C and Figure 7).

\section{Discussion}

The increase in the use of permethrin as a pesticide to manage household insects that constitute nuisance and pose health risk such as malaria is alarming [26, 27]. Despite being a very effective insecticide/pesticide [28, 29], there are increasing evidences on the toxicity tendency of permethrin [30-32]. Several studies have noted the health hazard associated with uncontrolled exposure to permethrin ranging from lymphocyte toxicity to teratogenesis [33, 34]. However, there is paucity of information on its neurotoxicity and behavioral manifestation following exposure to permethrin. This study characterized the effects of permethrin on body weight changes, oxidative stress, lipid peroxidation, histomorphology and Nissl profile of the hippocampus, prefrontal and cerebellar cortices.

Permethrin exposure induced a dose-dependent reduction in body weight in experimental animals. Although the exact mechanism through which permethrin causes weight loss is not fully clear, it has been suggested that the weight loss can possibly be associated with increase basal metabolic rate in the animals [35]. However, in the current study, there was increase in the weights of the brain areas assessed in the treated animals. Due to the insult on the brain following permethrin neurotoxicity, there is an increase in the metabolic activity of astrocytes which normally respond to brain injury as a form of defense mechanism [36]. Astrocytes become activated and undergo morphological changes, including increased cellular proliferation and hypertrophy (reactive astrogliosis). This increase in cell mass and size in the brain regions could contribute to the weight increase observed in the brain.

Exposure to permethrin perturbed the normal working and spatial memory indices in a differential manner. Animals exposed to permethrin presented with lower percentage correct alternation in the Y-maze and higher escape latency period in the Morris water maze. Furthermore, permethrin presented with anxiogenic properties. We deduced this 
Omotoso et al. I Permethrin exposure affects neurobehavior and cellular characterization

from the poor open field exploration and spending of longer time in the dark hole in the black and white box by animals exposed to permethrin. Locomotory activities of the permethrin-treated animals were not significantly altered, however, animals exposed to higher dose of permethrin had poorer locomotory index in the negative geotaxis behavioral paradigm. All these changes in the behavioral manifestation revealed that the key brain areas involved in processing and execution may have been compromised neurochemically or structurally, thereby affecting their normal physiology. There is a striking evidence that permethrin induces neuroinflammation in brain areas involved in memory processing, formation and consolidation [26]. An earlier study also established the ability of permethrin to induce inflammation, thereby affecting the normal physiology [31]. There is a direct link between neuroinflammation and cognitive decline [37-39]. This explains one of the likely mechanisms through which permethrin affects behavior.

Aside from neuroinflammation, oxidative stress has a direct association with cognitive decline [40, 41]. Hence, this study further investigated the neurotoxic tendencies of permethrin on the antioxidant status of brain regions using SOD, GPx and CAT as biomarkers. These enzymes are in the frontline of antioxidant defense system [42, 43]. Findings showed that permethrin affected the activities of these enzymes in a dose-dependent pattern across the three brain regions, such that the higher the dose of permethrin, the lower the enzyme activities. The implication of this is that permethrin induces oxidative stress thereby exacerbating the levels of reactive singlet and triplet oxygen species [42, 44-46]. This finding suggests that permethrin mediated its neurotoxicity by enhancing the generation of these reactive oxygen species to a point where the intrinsic antioxidant defense system becomes overwhelmed and ultimately compromised [47-49]. Several studies have previously underlined the toxicity of permethrin through oxidative stress [50-54]. As stated by Gargouri et al. [26], permethrin induces neuronal damage, changes redox state, and causes neuroinflammation in the hippocampus. One of the resultant effects of excessive reactive oxygen species (ROS) generation is lipid peroxidation [55]. The assessment of MDA, a by-product of lipid peroxidation, revealed an increase in the level of this metabolite in all three brain regions. Excess reactive species scavenge electrons from the polyunsaturated lipid bilayer of the neuronal membrane thereby compromising the structural integrity of cells within the brain and consequently initiates a cascade of chemical events that result into cell death and ultimately neurodegeneration [56, 57].

Although permethrin was administered for a 14-day period in this study, morphological alterations were observed in the hippocampus and prefrontal and cerebellar cortices. Some of these changes, including the degenerative and chromatolytic changes, were the consequence of assaults to cells and tissues of different brain regions arising from neurochemical and oxidative disruptions. The compromised structural integrity of the prefrontal cortex, hippocampus and cerebellum is capable of affecting vital neurologic functions of these brain regions leading to behavioral deficits.

Reactive astrocytosis is an expected phenomenon following brain injury [36]. All brain regions assessed demonstrated significant astrocytic reactivity with high dose permethrin, but of note is the marked immunoreactivity recorded in the prefrontal and cerebellar cortices. Deterioration in cognitive functions is a hallmark of synaptic pathology seen in several neurologic disorders, such as Alzheimer's disease, amyotrophic lateral sclerosis and Parkinson's disease [58-60], and oxidative stress and lipid peroxidation have been identified as part of a common pathophysiology of these neurodegenerative conditions [11,59]. The findings above correlated well with the depressed endogenous oxidative status that followed permethrin toxicity, and the oxidative stress has been suggested to be part of the pathophysiology of neurologic conditions associated with neurotoxicity [59].

\section{Conclusions}

Permethrin toxicity causes cognitive deficits and remarkable alteration in brain morphology and neurochemistry by inducing oxidative damage and lipid peroxidation. Hence, much caution should be exercised when used for any purpose to avoid contact.

\section{Funding}

This research did not receive any specific grant from funding agencies in the public, commercial, or not-for profit sectors

\section{Conflict of interest}

Authors declare no conflicts of interest.

\section{CRediT author statement}

GO: Conceptualization, Methodology, Supervision, Writing- Reviewing and Editing; OO: Methodology, Data curation, Writing- Original draft preparation, Investigation; SL: Methodology, Data curation, Writing- Original draft preparation, Investigation; IG: Writing- Reviewing and Editing; NM Writing- Original draft preparation; FA: Writing- Original draft preparation; AB1: Writing- Original draft preparation; AB2: Writing- Original draft preparation; BA: Writing- Original draft preparation; NA: Writing- Reviewing and Editing.s 


\section{ORCID}

Gabriel Omotoso : 0000-0001-7727-6943

\section{References}

[1] Chrustek A, Hołyńska-Iwan I, Dziembowska I, Bogusiewicz J, Wróblewski M, Cwynar A, et al. Current research on the safety of pyrethroids used as insecticides. Medicina (Kaunas, Lithuania) 2018;54(4):61.

[2] Soderlund DM. Molecular mechanisms of pyrethroid insecticide neurotoxicity: recent advances. Arch Toxicol. 2012;86(2):165-81.

[3] Keystone JS, Kozarsky PE, Freedman DO, Nothdruft HD, Connor BA. Travel medicine. 3rd ed. Elsevier; 2013.

[4] Nasuti C, Fattoretti P, Carloni M, Fedeli D, Ubaldi M, Ciccocioppo R, et al. Neonatal exposure to permethrin pesticide causes lifelong fear and spatial learning deficits and alters hippocampal morphology of synapses. J Neurodev Disord 2014;6:7.

[5] Banks SD, Murray N, Wilder-Smith A, Logan JG. Insecticide-treated clothes for the control of vector-borne diseases: a review on effectiveness and safety. Med Vet Entomol 2014;28(Suppl 1):14-25.

[6] Orsborne J, Deraedt Banks S, Hendy A, Gezan SA, Kaur H, Wilder-Smith A, et al. Personal protection of permethrin-treated clothing against Aedes aegypti, the vector of Dengue and Zika virus, in the laboratory. PLoS One 2016;11(5):e0152805.

[7] Kimani EW, Vulule JM, Kuria IW, Mugisha F. Use of insecticide-treated clothes for personal protection against malaria: a community trial. Malar J 2006;5:63.

[8] Baselt R. Disposition of toxic drugs and chemicals in man. 8th ed. Biomedical Publications; 2008. ISBN 978-09626523-7-0.

[9] Van Der Rhee HJ, Farquhar JP, Vermeulen NP. Efficacy and transdermal absorption of permethrin in scabies patients. Acta Derm Venereol 1989;69(2):170-3.

[10] Tornero-Velez R, Davis J, Scollon EJ, Starr JM, Setzer RW, Goldsmith MR, et al. A pharmacokinetic model of cisand trans-permethrin disposition in rats and humans with aggregate exposure application. Toxicol Sci 2012;130(1):33-47.

[11] Singh AK, Tiwari MN, Prakash O, Singh MP. A current review of cypermethrin-induced neurotoxicity and nigrostriatal dopaminergic neurodegeneration. Curr Neuropharmacol 2012;10(1):64-71.

[12] Crofton KM, Reiter LW. The effects of type I and II pyrethroids on motor activity and the acoustic startle response in the rat. Fundam Appl Toxicol 1988;10:624-634.

[13] Vijverberg HPM, van den Bercken J. Neurotoxicological effects and the mode of action of pyrethroid insecticides. Crit Rev Toxicol 1990;21:105-126.

[14] Bradberry SM, Cage SA. Proudfoot AT, Vale JA. Poisoning due to pyrethroids. Toxicol Rev 2005;24:93-106.

[15] Aldridge WN. An assessment of the toxicological properties of pyrethroids and their neurotoxicity. Crit Rev Toxicol 1990;21:89-104.

[16] Omotoso GO, Onanuga IO, Ibrahim RB. Histological effects of permethrin insecticide on the testis of adult Wistar rats. Ibnosina J Med BS 2014;6(3):125-129.

[17] Nunez J. Morris water maze experiment. J Vis Exp 2008;9(9):89-97.

[18] Momeni S, Segerström L, Roman E. Supplier-dependent differences in intermittent voluntary alcohol intake and response to naltrexone in Wistar rats. Front Neurosci 2015;9:424.

[19] Seibenhener ML, Wooten MC. Use of the open field maze to measure locomotor and anxiety-like behavior in mice. J Vis Exp 2015;(96):e52434. 
[20] Costall B, Jones BJ, Kelly ME, Naylor RJ, Tomkins DM. Exploration of mice in a black and white test box: Validation as a model of anxiety. Pharmacol Biochem Behav 1989;32(3):777-785.

[21] Fedele G, Green EW, Rosato E, Kyriacou CP. An electromagnetic field disrupts negative geotaxis in Drosophila via a CRY-dependent pathway. Nat Commun 2014;5:4391.

[22] Fallone CA, Barkun A, Loo V, Wickham C, Hu X, Kostyk R. Hematoxylin and eosin staining is sufficient for diagnosis of Helicobacter pylori infection. Gastroenterol 1995;108(4 Supplement 1):A92.

[23] Kádár A, Wittmann G, Liposits Z, Fekete C. Improved method for combination of immunocytochemistry and Nissl staining. J Neurosci Methods 2009;184:115-8.

[24] Acs P, Kalman B. Pathogenesis of multiple sclerosis: what can we learn from the cuprizone model. Methods Mol Biol 2012;900:403-31.

[25] Thiha A, Ibrahim F. A colorimetric enzyme-linked immunosorbent assay (ELISA) detection platform for a pointof-care Dengue detection system on a lab-on-compact-disc. Sensors (Basel) 2015;15(5):11431-11441.

[26] Gargouri B, Yousif NM, Attaai A, Bouchard M, Chtourou Y, Fiebich BL, et al. Pyrethroid bifenthrin induces oxidative stress, neuroinflammation, and neuronal damage, associated with cognitive and memory impairment in murine hippocampus. Neurochem Int 2018;120:121-133.

[27] Shishovska MA, Stefova MT. Fast and universal HPLC method for determination of permethrin in formulations using 1.8- $\mu \mathrm{m}$ particle-packed column and performance comparison with other column types. J Chromatogr Sci 2012;50(1):43-50.

[28] Prater MR, Gogal JR, Blaylock BL, Longstreth J, Holladay SD. Single-dose topical exposure to the pyrethroid insecticide, permethrin in C57BL/6N mice: effects on thymus and spleen. Food Chem Toxicol 2002;40(12):18631873.

[29] Yoon KS, Gao JR, Lee SH, Coles GC, Meinking TL, Taplin D, et al. Resistance and cross-resistance to insecticides in human head lice from Florida and California. Pestic Biochem Physiol 2004;80(3):192-201.

[30] Choi J, Rose RL, Hodgson E. In vitro human metabolism of permethrin: the role of human alcohol and aldehyde dehydrogenases. Pestic Biochem Physiol 2002;74:117-128.

[31] Fedeli D, Carloni M, Nasuti C, Gambini A, Scocco V, Gabbianelli R. Early life permethrin exposure leads to hypervitaminosis D, nitric oxide and catecholamines impairment. Pestic Biochem Physiol 2013;107(1):93-97.

[32] Zhang SY, Ueyama J, Ito Y, Yanagiba Y, Okamura A, Kamijima M, et al. Permethrin may induce adult male mouse reproductive toxicity due to cis isomer not trans isomer. Toxicol 2008;248(2-3):136-141.

[33] Dhivya Vadhana MS, Siva Arumugam S, Carloni M, Nasuti C, Gabbianelli R. Early life permethrin treatment leads to long-term cardiotoxicity. Chemosphere 2013;93(6):1029-1034.

[34] Wang X, Martínez MA, Dai M, Chen D, Ares I, Romero A, et al. Permethrin-induced oxidative stress and toxicity and metabolism. A review. Environ Res 2016;149:86-104.

[35] Falcioni ML, Nasuti C, Bergamini C, Fato R, Lenaz G, Gabbianelli R. The primary role of glutathione against nuclear DNA damage of striatum induced by permethrin in rats. Neurosci 2010;168(1):2-10.

[36] Sofroniew MV. Molecular dissection of reactive astrogliosis and glial scar formation. Trends Neurosci 2009;32(12):638-647.

[37] Barrientos RM, Hein AM, Frank MG, Watkins LR, Maier SF. Intracisternal interleukin-1 receptor antagonist prevents postoperative cognitive decline and neuroinflammatory response in aged rats. J Neurosci 2012;32(42):14641-14648.

[38] Terrando N, Eriksson LI, Ryu JK, Yang T, Monaco C, Feldmann M, et al. Resolving postoperative neuroinflammation and cognitive decline. Ann Neurol 2011;70(6):986-995.

[39] Vacas S, Degos V, Feng X, Maze M. The neuroinflammatory response of postoperative cognitive decline. Br Med Bull 2013;106:161-178. 
[40] Liu J, Head E, Gharib AM, Yuan W, Ingersoll RT, Hagen TM, et al. Memory loss in old rats is associated with brain mitochondrial decay and RNA/DNA oxidation: partial reversal by feeding acetyl-L-carnitine and/or R-alpha -lipoic acid. Proc Natl Acad Sci USA 2002;99(4):2356-2361

[41] Pietá Dias C, De Lima MM, Presti-Torres J, Dornelles A, Garcia VA, Scalco FS, et al. Memantine reduces oxidative damage and enhances long-term recognition memory in aged rats. Neurosci 2007;146(4):1719-1725.

[42] Halliwell B. Oxidative stress and neurodegeneration: where are we now? J Neurochem 2006;97(6):1634-1658.

[43] Omotoso GO, Gbadamosi IT, Afolabi TT, Abdulwahab AB, Akinlolu AA. Ameliorative effects of Moringa on cuprizone-induced memory decline in rat model of multiple sclerosis. Anat Cell Bio 2018a;51:119-127.

[44] Farombi EO, Owoeye O. Antioxidative and chemopreventive properties of Vernonia amygdalina and Garcinia biflavonoid. Int J Environ Res Public Health 2011;8:2533-2555.

[45] Federico A, Cardaioli E, Pozzo P, Formichi P, Gallus GN, Radi E. Mitochondria, oxidative stress and neurodegeneration. J Neurol Sci 2012;322(1-2):254-262.

[46] Gbadamosi IT, Omotoso GO, Olajide OJ, Dada-Habeeb SO, Arogundade TT, Lambe E, et al. Moringa protects against nicotine-induced morphological and oxidative damage in the frontal cortex of Wistar rats. Anatomy 2016;10:170-176.

[47] Gilgun-Sherki Y, Melamed E, Offen D. Oxidative stress induced-neurodegenerative diseases: the need for antioxidants that penetrate the blood brain barrier. Neuropharmacol 2001;40(8):959-975.

[48] Habtemariam S. The African and Arabian moringa species: chemistry, bioactivity and therapeutic applications. Elsevier. 2017.

[49] Matés JM, Pérez-Gómez C, Núñez DE, Castro I. Antioxidant enzymes and human diseases. Clin Biochem 1999;32(8):595-603.

[50] Barrueco C, Herrera A, Caballo C, De La Peña E. Cytogenetic effects of permethrin in cultured human lymphocytes. Mutagen 1992;7(6):433-437.

[51] Türkez H, Toğar B. Olive leaf extract counteracts genotoxicity and oxidative stress of permethrin in human lymphocytes. J Toxicol Sci 2011;36(5):531-537.

[52] Gong Y, Li T, Zhang L, Gao X, Liu N. Permethrin induction of multiple cytochrome P450 genes in insecticide resistant mosquitoes, Culex quinquefasciatus. Int J Biol Sci 2013;9(9):863-871.

[53] Jin Y, Liu J, Wang L, Chen R, Zhou C, Yang Y, et al. Permethrin exposure during puberty has the potential to enantioselectively induce reproductive toxicity in mice. Environ Int 2012 42:144-151.

[54] Lidova J, Stara A, Kouba A, Velisek J. The effects of cypermethrin on oxidative stress and antioxidant biomarkers in marbled crayfish (Procambarus fallax f. virginalis). Neuro Endocrinol Lett 2016;37(Suppl 1):53-59.

[55] Wang P, Xie K, Wang C, Bi J. Oxidative stress induced by lipid peroxidation is related with inflammation of demyelination and neurodegeneration in multiple sclerosis. Eur Neurol 2014;72(3-4):249-254.

[56] Omotoso Go, Gbadamosi IT, Olajide OJ, Dada-Habeeb SO, Arogundade TT, Yawson EO. Moringa oleifera phytochemicals protect the brain against experimental nicotine-induced neurobehavioral disturbances and cerebellar degeneration. Pathophysiol 2018b;25(1):57-62.

[57] Omotoso GO, Ukwubile II, Arietarhire L, Sulaimon F, Gbadamosi IT. Kolaviron protects the brain in cuprizoneinduced model of experimental multiple sclerosis via enhancement of intrinsic antioxidant mechanisms: Possible therapeutic applications? Pathophysiol 2018c;25(4):299-306.

[58] Naylor R, Hill AF, Barnham KJ. Is Covalently Cross-linked A $\beta$ responsible for synaptotoxicity in Alzheimer's disease? Curr Alzheimer Res 2008;5(6) DOI: 10.2174/156720508786898433.

[59] Lopachin RM, Gavin T, Barber DS. Type-2 alkenes mediate synaptotoxicity in neurodegenerative diseases. Neurotoxicol 2018;29(5):871-882. 
Omotoso et al. I Permethrin exposure affects neurobehavior and cellular characterization

[60] Farizatto KLG, Almeida, MF, Long RT, Bahr BA. Early synaptic alterations and selective adhesion signaling in hippocampal dendritic zones following organophosphate exposure. Sci Rep 2019;9:6532. 